\title{
Tumor fibroso solitario de la glándula tiroides con extensión intratorácica
}

\author{
Solitary fibrous tumor of the thyroid gland with intrathoracic extension \\ Carlos Simón Duque $\mathrm{F}^{1}$, Juliana Sierra $\mathrm{Z}^{2}$, Juan Fernando Muñoz $\mathrm{T}^{3}$, Wilfredy Castaño $\mathrm{R}^{4}$, \\ Andrés Zerrate $\mathbf{M}^{4}$, Alejandro Vélez $\mathrm{H}^{5}$.
}

\begin{abstract}
RESUMEN
El tumor fibroso solitario (TFS) es una neoplasia mesenquimal de células fusiformes infrecuente descrita inicialmente en la pleura pero con localización reconocida en otros sitios. El TFS de la glándula tiroides (TFST) es aun más raro. Se describe el caso de una mujer de 65 años de edad, con masa cervical de crecimiento progresivo, a tal punto que ya la masa se hacía notable en parte inferior de su cuello, siendo este el único síntoma narrado.

La paciente presentaba una gran masa tiroidea en el lóbulo derecho con extensión intratorácica. Se practicó hemitiroidectomia derecha (paciente tenia historia previa de resección del lóbulo tiroideo izquierdo por lesión benigna) Total, además de toracotomía endoscópica por la extensión de la masa la cual en la tomografía contrastada, se originaba en el lóbulo tiroideo derecho y descendía paralelo a la columna dorsal desplazando a la tráquea y el esófago. La patología reportó un tumor de 15 centímetros, con células fusiformes y patrón de crecimiento hemangiopericítico sin pleomorfismo, atipia, mitosis o necrosis. Luego de practicar diferentes estudios y marcadores tumorales, de todos estudios fueron positivos CD34, BCl2, CD99 y vimentina. Se diagnosticó tumor fibroso solitario de glándula tiroides. El nervio vago derecho fue lesionado en la cirugía, actualmente está en terapia de voz. Al momento actual casi seis meses luego de su cirugía, no se documenta recidiva tumoral.
\end{abstract}

Palabras claves: Bocio intratorácico, tumor fibroso solitario, tiroides.

\footnotetext{
Médico Cirugía Cabeza y Cuello Hospital Pablo Tobón Uribe, Medellín, Colombia.

2 Fellow Endocrinología Universidad de Antioquia, Colombia.

3 Médico Residente Otorrinolaringología IV año Universidad Militar Nueva Granada, Colombia.

4 Médico Tórax Hospital Pablo Tobón Uribe, Medellín, Colombia.

5 Médico, Anátomo-Patólogo Hospital Pablo Tobón Uribe, Medellín, Colombia.
}

Recibido el 24 de abril de 2016. Aceptado el 23 de mayo de 2016. 


\section{ABSTRACT}

Solitary fibrous tumor (SFT) is a rare mesenchymal neoplasm initially described as spindle cells in the pleura but recognized location elsewhere. The TFS thyroid gland (TFST) even infrequent. A case of a 65 years old woman with progressive growth of a right cervical mass without associated symptoms described other than cosmesis is described. The patient had a thyroid mass in the right lobe with intrathoracic extension. A right Hemi thyroidectomy and thoracotomy for the extent of the mass was performed. Pathology reported a $15 \mathrm{~cm}$ tumor with spindle cell and growth pattern hemangiopericitic without pleomorphism, atypia, mitosis or necrosis. Immunohistochemistry was positive for CD34, BCl-2, CD99 and vimentin, making the diagnosis of solitary fibrous tumor of thyroid gland. The patient's right Vagus nerve was injured intraoperatively and she is currently under voice therapy. Currently now, almost six months after her surgery she is free of disease.

Key words: Intrathoracic goiter, solitary fibrous tumor, thyroid.

\section{INTRODUCCIÓN}

El tumor fibroso solitario es un tumor raro de origen mesenquimal descrito por primera vez en pleura'. Desde entonces se ha documentado en múltiples órganos incluidos la glándula tiroides. En esta localización su diagnóstico diferencial incluye una amplia gama de patologías benignas y malignas de tejido estromal y epitelial tiroideo, y puede llegarse al diagnóstico definitivo por la inmunohistoquímica característica. Son pocos los casos descritos de tumor fibroso solitario tiroideo (TFST) en la literatura mundial y es el primero descrito en Colombia.

\section{CASO CLÍNICO}

Paciente femenina de 65 años quien consulta por masa cervical derecha indolora de cinco meses de evolución. No tenía otra sintomatología diferente a la producida por la alteración cosmética observable con abultamiento en la región supraesternal. Ocho años antes la paciente había sido sometida a hemitiroidectomía izquierda en otro hospital por patología benigna. La ecografía de cuello, demostró masa que ocupaba el lóbulo tiroideo derecho y descendía al tórax; el aspirado biopsia dirigido por ecografía fue reportado como Bethesda I, no concluyente. La tomografía contrastada de cuello y tórax confirmó una masa que se originaba en el lóbulo tiroideo derecho de $6 \times 15 \times 5 \mathrm{~cm}$, descendiendo al mediastino, desplazando la tráquea superior y torácica, al igual que la vena yugular interna y carótida común (Figuras 1 y 2).

La paciente fue intervenida en conjunto con cirugía de tórax. Se realizó videotoracoscopía

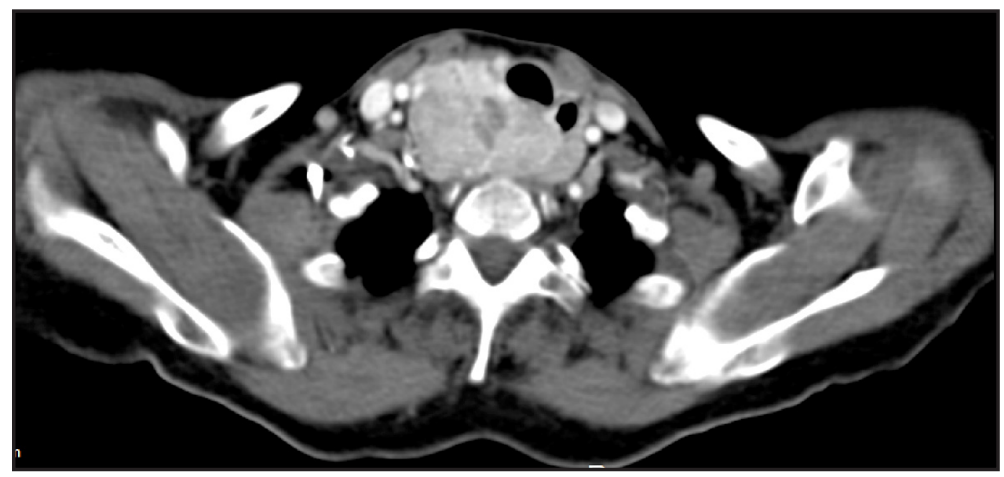

Figura 1. Tomografía computarizada contrastada cuello muestra tumor compresivo sobre tráquea cervical y esófago. 


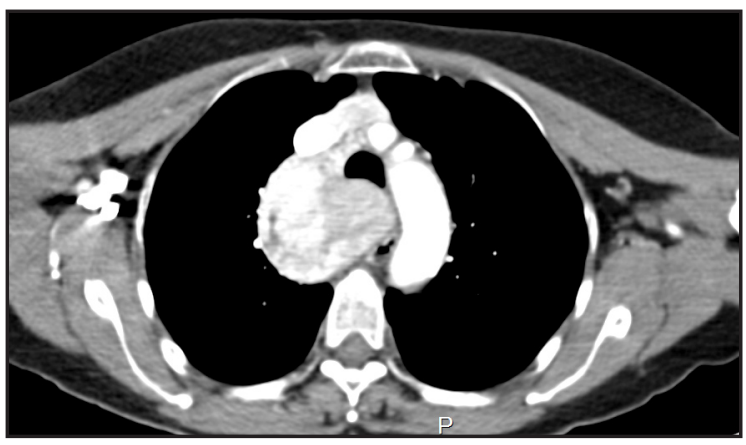

Figura 2. Tomografía computarizada muestra extensión del tumor hacia el mediastino.

para la liberación del componente tumoral intratorácico considerando su liberación posterior por cuello, sin ser factible dadas las múltiples adherencias del tumor, razón por lo cual se realizó toracotomía posterolateral. Posteriormente, se practicó hemitiroidectomía derecha, disección mediastinal con monitoreo intraoperatorio de nervio laríngeo $\left(\mathrm{NIM}^{\circledR} 3.0\right.$ Medtronic ${ }^{\circledR}$ JacksonviIle, Florida, USA), liberando el tumor en la parte superior. Durante la cirugía se encontró en cuello una masa adherida a músculos pretiroideos que ingresaba a mediastino posterior hasta región subcarinal con adherencias a nervio laríngeo recurrente, tráquea, esófago, vena ácigos y vena cava superior al igual que a la pleura parietal costal y mediastinal. Al momento de abordar el nervio vago, no se observa señal en el monitor, indicando posible lesión intratorácica del nervio durante el proceso de liberación y remoción del tumor. Sangrado intraoperatorio aproximado 1.500 cc. La paciente no presentó otras complicaciones en el posoperatorio. Al momento, casi seis meses luego de la cirugía, la paciente está en el proceso de rehabilitación de su parálisis de la cuerda vocal derecha, si evidencia de recurrencia tumoral.

\section{PATOLOGÍA}

Al microscopio los cortes histológicos muestran una neoplasia fusocelular en patrón hemangiopericítico sin pleomorfismo, atipia, mitosis o necrosis. Los marcadores de inmunohistoquímica de tipo CD34, Bcl2, CD99 y vimentina son positivos. TTF1, calcitonina, desmina y $\mathrm{S} 100$ son negativos. Por lo anterior se realiza diagnóstico tumor fibroso solitario (Figuras 3 y 4 ).

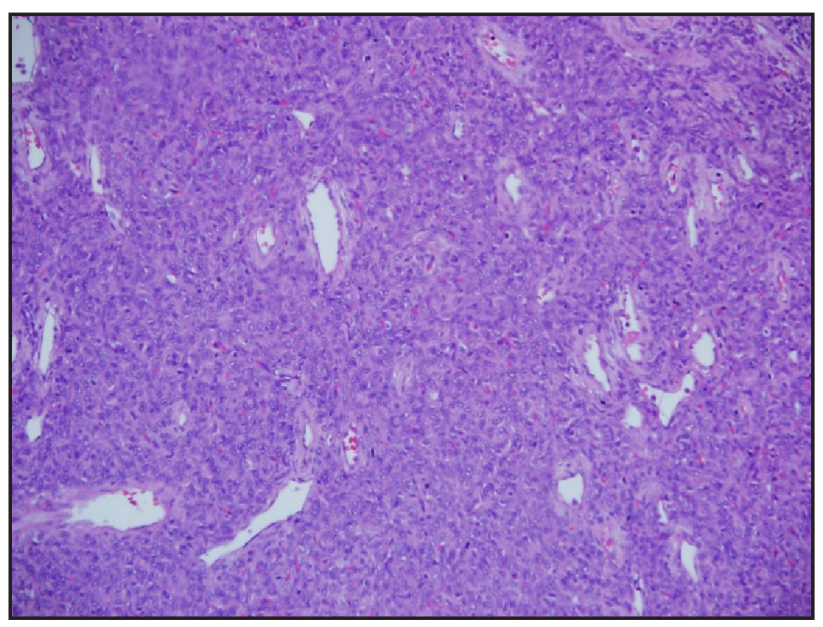

Figura 3. Proliferación de células fusiformes en patrón hemangiopericítico sin pleomorfismo, atipia, mitosis o necrosis. Tinción hematoxilinaeosina. 


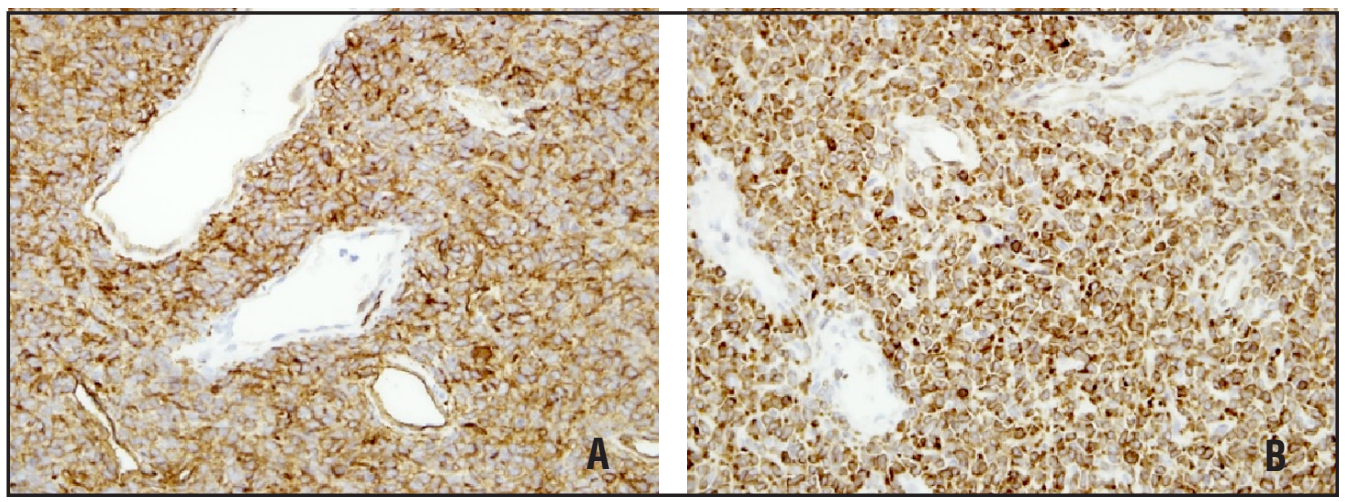

Figura 4. Tinción inmunohistoquimica CD34 (citoplasmática) A, y Bcl2 (citoplasmática y nuclear) B, positivas.

\section{DISCUSIÓN}

El tumor fibroso solitario (TFS) es un tumor de origen mesenquimal descrito por primera vez por Klemperer y Robin ${ }^{1}$ en 1931, haciendo referencia a una neoplasia de células fusiformes localizada en la pleura. Desde entonces, hay reportes de localización en otros sitios incluyendo cavidad nasal, cavidad oral, mama, glándula parótida, epiplón, riñones, tracto gastrointestinal, hígado y extremidades². EI TFS de la glándula tiroides (TFST) es raro, a la fecha se han descrito 35 casos con el presente ${ }^{3-24}$. Con los datos disponibles, el TFST tiene una edad media de presentación de 58 años (rango 28-88 años), sin diferencia de género, y con tamaño variable entre 17 y $150 \mathrm{~mm}$; siendo este caso el tumor de mayor tamaño reportado. Se localizan con igual frecuencia en ambos lóbulos y puede tener extensión intratorácica ${ }^{17,22}$, como se ha reportado en dos casos más. La clínica es similar a la reportada por otras neoplasias tiroideas, masa cervical firme, de crecimiento progresivo, en ocasiones significativo, produciendo síntomas como disnea, disfonía y disfagia. Las pruebas de función tiroidea por lo general son normales. Las imágenes de tomografía y ecografía muestran un tumor de apariencia heterogénea, con 0 sin formación de nódulos o quistes en su interior. Las características histológicas son la proliferación de células fusiformes o similares a fibroblastos y tejido conectivo en proporciones variables. Dichas células pueden adoptar diferente arquitectura. El patrón "sin patrón" y el hemangiopericítico (patrón de este caso) son los más comunes. Otros menos frecuentes son arremolinado, ondulado, desmoide, y en empalizada. Hay poca o moderada celularidad y la actividad mitótica es escasa. Se considera que el TFST es de origen mesenquimatoso y la inmunohistoquímica es usualmente positiva para CD34, CD99, BCL2, vimentina. Marcadores como CD5, citoqueratina, factor de transcripción tiroideo 1 (TTF1), calcitonina, sinaptofisina, cromogranina A, y desmina, son generalmente negativos. Existe además una variante adipocítica del TFST que muestra positividad para la proteína $S 100$ en los focos lipomatosos. No hay criterios de malignidad propiamente establecidos para el TFST. Se han propuesto criterios de comportamiento más agresivo en TFS de otros sitios ${ }^{25}$ : alta celularidad, atipia citológica, mayor frecuencia de mitosis ( $>4 / 10$ HPF), evidencia de necrosis tumoral 0 márgenes infiltrantes. En la literatura hay reporte de dos casos de TFST ${ }^{16,24}$ que cumplen con estas características, uno de ellos con recurrencia y enfermedad metastásica a los 5 meses de seguimiento.

El diagnóstico diferencial de TFST incluye tumores mesenquimales de células fusiformes benignos y malignos, como tumores de músculo liso y tumores de la vaina de nervio periférico, y tumores epiteliales con células de morfología fusiforme como tumor epitelial fusiforme con diferenciación similar al timo (SETTLE, por sus siglas en inglés), carcinoma que muestra diferenciación similar al timo (CASTLE, por sus siglas en inglés), carcinoma medular, carcinoma papilar variante similar a 
fascitis, carcinoma no diferenciado (anaplásico), adenoma folicular con células fusiformes, bocio con células fusiformes, y tiroiditis de Riede ${ }^{26}$.

El tratamiento del TFS de tiroides consiste principalmente en la resección quirúrgica, procedimiento que a la vez permite el diagnóstico histológico definitivo. $Y$ aunque ocasionalmente, existe el riesgo de recidiva local y de metástasis a distancia,

\section{BIBLIOGRAFÍA}

1. Klemperer P, Rabin CB. Primary neoplasms of the pleura: a report of five cases. Arch Pathol 1931; 11: 385-412.

2. Brunnemann R, Ro J, Ordonez N, Mooney J, ElNaggar AK, AyALA AG. Extrapleural solitary fibrous tumor: a clinicopathologic study of 24 cases. Mod Pathol 1999; 12(11): 1034-42.

3. Taccagni G, Sambade C, Nesland J, Terreni MR, Sobrinho- Simo Es M. Solitary fibrous tumor of the thyroid: clinicopathological, immunohistochemical and ultrastructural study of three cases. Virchows Arch A Pathol Anat Histopathol 1993; 422(6): 491-7.

4. Cameselle-Teijeiro J, Varela-Duran J, Fonseca E, Villanueva JP, Sobrinho-Simoes M. Solitary fibrous tumor of the thyroid. Am J Clin Pathol 1994; 101: 535-8.

5. Kie J, Kim J, Park Y, Lee MK, Yang WI, Park JS. Solitary fibrous tumor of the thyroid. Histopathology 1997; 30:365-8.

6. Rodríguez I, Ayala E, Caballero C et al. Solitary fibrous tumor of the thyroid gland: report of seven cases. Am J Surg Pathol 1997; 25: 14248.

7. Deshmukh N, Mangham D, Warfield A, Watkinson JC. Solitary fibrous tumor of the thyroid gland. $J$ Laryngol Otol 2001; 115(11): 940-2.

8. Bohorquez CL, González-Campora R, Loscertales MC, Escudero AG, Mezquita JC. Solitary fibrous tumor of the thyroid with capsular invasion. Pathol Res Pract 2003; 199: 687-90.

9. Parwani AV, Galindo R, Steinberg DM, Zeiger MA, Westra WH, Alı SZ. Solitary fibrous tumor of the thyroid: cytopathologic findings and differential diagnosis. Diagn Cytopathol 2003; 28: 213-6. la resección quirúrgica completa y el seguimiento cuidadoso son esenciales para estos pacientes. La terapia de supresión de TSH con hormonas no tiene ninguna razón de ser, pues el origen de las células tumorales es estromal. Dados los escasos reportes de TFST malignos, no hay datos sobre modalidades terapéuticas como quimioterapia y radioterapia.

10. Babouk NL. Solitary fibrous tumor of the thyroid gland. Saudi Med J 2004; 25: 805-7.

11. Tanahashi J, Kashima K, Daa T, et al. Solitary fibrous tumor of the thyroid gland: report of two cases and review of the literature. Pathol Int 2006; 56: 471-7.

12. Papi G, Corrado S, Ruggiero C, Livolsi VA. Solitary fibrous tumor of the thyroid gland associated with papillary thyroid carcinoma. Thyroid 2006; 16: 319-20.

13. Papi G, Corrado S, Uberti ED, Rotı E. Solitary fibrous tumor of the thyroid gland. Thyroid 2007; 17: 119-26.

14. Santeusanio G, Schiaroli S, Ortenzi A, Mule A, Perrone G, Fadda G. Solitary fibrous tumour of thyroid: report of two cases with immunohistochemical features and literature review. Head Neck Pathol 2008; 2: 231-5.

15. Farrag TY, Micchelli S, Tufano RP. Solitary fibrous tumor of the thyroid gland. Laryngoscope 2009; 119: 2306-8.

16. Ning S, Song X, Xiang L, Chen Y, Cheng Y, Chen $\mathrm{H}$. Malignant solitary fibrous tumor of the thyroid gland: report of a case and review of the literature. Diagn Cytopathol 2010; 39: 694-9.

17. Larsen SR, Godballe C, Krogdahl A. Solitary fibrous tumor arising in an intrathoracic goiter. Thyroid 2010; 20: 435-7.

18. Song Z, Yu C, Song X, Wei L, Liu A. Primary solitary fibrous tumor of the thyroid - report of a case and review of the literature. J Cancer 2011; 2: 206-9.

19. Verdi D, Pennelli G, Pelizzo MR, Toniato A. Solitary fibrous tumor of the thyroid gland: a report of two cases with an analysis of their clinical and pathological features. Endocr Pathol 2011; 22: 165-9. 
20. Gallegos I, Concha C, Ferrada C. Tumor fibroso solitario del tiroides. Revista Chilena de Cirugía 2012; 64(3): 285-8.

21. López-García PL, González M ÁM, Egea AS, Díaz ES, López IQ, Barrios AP. Tumor fibroso solitario primario en tiroides: comunicación de un caso y revisión de la literatura. Revista Española de Patología 2013; 46(4): 261-4.

22. Lin MW, Wu CT, Lee YH, Chang YL. Intrathoracic Thyroid Solitary Fibrous Tumor Presenting with Respiratory Failure. Annals of Thoracic and Cardiovascular Surgery 2014; 20: 427-9.

23. Mizuuchi Y, Yamamoto $H$, Nakamura K, Shirahane K, Souzaki M, Tanaka M, Oda Y. Solitary fibrous tumor of the thyroid gland. Med Mol Morphol 2014; 47: 117-22.
23. Alves Filho W, Mahmoud RRdgl, Ramos D, Araujo-Filho VJFD, Lima PPD, Cernea C, BRANDÃo L. Malignant solitary fibrous tumor of the thyroid: a case-report and review of the literature. Arq Bras Endocrinol Metab 2014; 58(4): 402-6.

24. Vallat-Decouvelaere AV, Dry SM, Fletcher CD. Atypical and malignant solitary fibrous tumors in extrathoracic locations: evidence of their comparability to intra-thoracic tumors. The American Journal of Surgical Pathology 1998; 22(12): 1501-11.

25. Papı G, Corrado S, LiVolsı VA. Primary spindle cell lesions of thyroid gland. An overview. Am J Cli Pathol 2005; 124: S95-S123. 